\title{
Erwartungen der Privaten Krankenversicherung an eine Strukturreform der Krankenversicherung
}

\author{
von Heinrich Frommknecht*
}

\section{Notwendigkeit und Ziel einer Strukturreform}

Unser Gesundheitswesen leidet. Kranksein droht, unbezahlbar zu werden. Die Lücke zwischen Ausgaben und Einnahmen der gesetzlichen Krankenversicherung (GKV) wird immer größer, 1984 lag das Defizit bereits bei rd. 3 Mrd. DM. Bei gleichbleibender Kostenentwicklung würde nach Angaben der GKV das Defizit bis zum Jahr 2000 auf 70 Mrd. DM anwachsen, der Beitragssatz in der GKV auf 17\% ansteigen (zum Vergleich : 1970 lag er noch bei rund $8 \%$ ). Würde diese Vision Realität, käme unser System der sozialen Sicherung ernsthaft in Gefahr - zumal die Belastungen auch der gesetzlichen Rentenversicherung eher noch stärker werden.

Besonders bedenklich ist, daß der Kostenexplosion keine gleichgerichtete "Nutzenexplosion" gegenüberstand. Im Gegenteil : In der Bundesrepublik Deutschland fielen 1985 je Arbeitnehmer statistisch gesehen 128 Arbeitsstunden wegen Krankheit aus, dagegen in den USA weniger als die Hälfte, nämlich 62 und in Japan lediglich 34. Sind die Deutschen wirklich gesundheitlich soviel mehr gefährdet? Oder ist diese Entwicklung vielmehr nicht auch die Folge von sozialgesetzlichen Möglichkeiten, die wenig verantwortungsbewußt ausgenutzt werden?

\subsection{Interventionen schaffen keine Abhilfe}

Die Entwicklung ist auch deshalb so besorgniserregend, weil der bisher betriebenen sogenannten Kostendämpfungspolitik im Gesundheitswesen kein nachhaltiger Erfolg beschieden war. Es wurde versucht, punktuell "per Verordnung" Kosten zu dämpfen. Verschiedene Kostendämpfungsgesetze wurden verabschiedet. Sie haben jedoch weniger Kosten gedämpft als weiteren Interventionsbedarf geschaffen.

Uberlegungen $\mathrm{zu}$ einer notwendigen Strukturreform des Krankenversicherungssystems sind aber nicht nur Sache der GKV als unmittelbar Betroffene, sondern ebenso auch Aufgabe der privaten Krankenversicherung (PKV) in ihrer Funktion als Alternative und Ergänzung zur GKV.

* Chairman of the board of Management, Signal Krankenversicherung AG, Dortmund; paper presented at the 14a General Assembly of the Geneva Association, Berlin, June 15, 1987. 


\section{Zu den Ursachen der Kostenexplosion}

Das Wirtschaftssystem der Bundesrepublik Deutschland ist in seinen Grundzügen marktwirtschaftlich organisiert, im allgemeinen steuern Preise als Indikatoren für Knappheit die Verhaltensweisen der Marktteilnehmer und veranlassen sie, sparsam mit Gütern umzugehen. Für das Gesundheitswesen und die GKV gelten jedoch andere Regeln. Die Versorgung mit Gesundheitsgütern wird als öffentliche Aufgabe angesehen. Die Erfüllung dieser Aufgaben wird von staatsähnlichen Organisationen wahrgenommen, die zu ihrer Finananzierung auf das Mittel des Zwangs zurückgreifen können (GKV!). Dazu kommt der öffentlich rechtliche Status von Institutionen des Gesundheitswesens. Das Gesundheitssystem, wie wir es heute vorfinden, ist demzufolge dem öffentlichen Sektor zuzurechnen.

\subsection{Zuviel gesetzlich Versicherte}

Dies ergibt sich auch daraus, daß heute knapp $90 \%$ der Bevölkerung gesetzlich krankenversichert sind. Die GKV wurde im Laufe der Zeit immer mehr für die verschiedensten Bevölkerungs- und Einkommensschichten geöffnet. Dadurch wurde der Raum für private Vorsorge eingeengt. Durch verschiedene gesetzgeberische Maßnahmen hatte die PKV einen Rückgang ihrer Vollversicherten innerhalb des Zehnjahreszeitraums 1964 bis 1974 von 6,7 Mio. auf 4,2 Mio. Personen zu verzeichnen. Das waren-37\%. Inzwischen hat sich dieser verhängnisvolle Trend jedoch wieder umgekehrt, heute verzeichnet die PKV rund 5,36 Mio. Vollversicherte.

Die Ausweitung der gesetzlichen Krankenversicherung ist deshalb bedenklich, weil sie ursprünglich als Solidareinrichtung für sozial Schutzbedürftige errichtet worden ist. Deshalb genießt sie auch heute noch verschiedene Privilegien, wie Steuerfreiheit, Kassenrabatt beim Bezug von Arzneimitteln, Beitragseinzug durch die Arbeitgeber etc. Es sind ernste Zweifel angebracht, ob die Struktur unseres Krankenversicherungssystems unserem erreichten hohen Wohlstandsniveau noch gerecht wird. Eigentlich sollte man annehmen, daß mit steigendem Wohlstand immer mehr Leute in die Lage versetzt werden, Eigenvorsorge auch im Krankheitsfall zu treffen. Dafür haben wir doch eine leistungsfähige PKV! Der Trend ging jedoch - wie gezeigt - genau in die entgegengesetzte Richtung.

\subsection{Fehlende Anreize zu sparsamem Verhalten}

Ursächlich für Kostenexplosion ist auch die Mentalität eines großen Teils der Versicherten. Sie meinen, sie müßten ihre immer stärker steigenden Beiträge, die sie an anonyme Solidargemeinschaften entrichten, wieder "hereinholen" und verlangen kostspieligere und längere Behandlung als vielleicht nötig wäre. Sie stoßen dabei kaum auf Widerstand, denn der Arzt wird nicht für das Endprodukt "Gesundheit" entlohnt, sondern für Zwischenprodukte, also einzelne Gesundheitsleistungen. Das ungebremste Anspruchsdenken wird dabei leider oft mit wachsendem Gesundheitsbewußtsein gleichgesetzt : Der Ansturm von Bagatellfällen in den Arztpraxen ist größtenteils auf dieses Denken zurückzuführen. Die "Abfertigung" von 50 bis 60 Patienten pro Tag, also die sogenannte 5 MinutenMedizin, ist für den Kassenarzt die Regel.

\subsection{Mangelnde Kostentransparenz}

Der Versicherte hat keinen Überblick über das Verhältnis von Leistung und Gegenleistung - er bekommt die Abrechnungen nicht zu Gesicht, erfährt also auch nicht, was die Wiederherstellung seiner Gesundheit kostet. So wird das Versicherungsprinzip in ein reines 
Versorgungsprinzip transformiert. Ein weiteres Indiz für fehlendes Wirtschaftlichkeitsinteresse und damit Verschwendung im Gesundheitsbereich sind die gehorteten Arzneimittel, die nicht verbraucht und schließlich weggeworfen werden. Es sind dies jährlich schätzungsweise Medikamente im Werte von ca. 2 Mrd. DM.

Die Möglichkeiten für den Wettbewerb sind im Sozialversicherungssystem naturgemäß nur beschränkt, wenn das Prinzip der Solidarität nicht gefährdet werden soll. Soweit aber dennoch Wettbewerb möglich ist, hat er sich in der Vergangenheit leider nur als kostentreibender Leistungswettbewerb dargestellt. Dadurch sollten vor allem gut verdienende Angestellte gewonnen werden. Durch die zusätzlichen Leistungsangebote wurden wiederum die Ausgaben erhöht. Auch hier gibt es also kaum Ansätze zur Ausgabenbegrenzung und damit Kostendämpfung.

\section{Erwartungen der PKV}

Die Erwartungen an eine Strukturreform gehen auseinander, je nachdem, ob marktwirtschaftliche Öberlegungen mit der Forderung nach mehr Subsidiarität im Vordergrund stehen, oder ob man sich mehr am öffentlich rechtlichen Solidarsystem ausrichtet. Die PKV fordert mehr Subsidiarität, also mehr eigenverantwortliche Vorsorge, denn Sozialpolitik darf kein Selbstzweck sein. Mehr Subsidiarität muß jedoch nicht weniger Solidarität zu Folge haben. Denn Solidarität beschränkt sich nämlich nicht nur auf einen finanziellen Solidarausgleich, sondern bedeutet auch Leistungs- und Interessenverzicht derjenigen, die Fremdhilfe nicht nötig haben. Die Frage lautet also : Wo kann durch mehr Eigenverantwortung und mehr private Vorsorge die Wirksamkeit der medizinischen Versorgung verbessert werden und wo wäre mehr Solidarität angebracht? Bundesarbeitminister Dr. Blüm hat in seinen zehn Grundsätzen für ein gesundheitspolitisches Gesamtkonzept die Weichen richtig gestellt, nämlich die gesetzliche Krankenversicherung als Solidareinrichtung auf das zu beschränken, was sozial- und gesundheitspolitisch wirklich nötig ist.

\subsection{GKV auf wirklich Schutzbedürftige begrenzen}

Sozial- und gesundheitspolitisch sicherlich nicht nur nicht nötig, sondern heute nahezu unvertretbar ist, daß fast $90 \%$ der Bevölkerung als vermeintlich sozial Schutzbedürftige in der GKV versichert sind. Mehr Eigenverantwortung muß also bedeuten, die gesetzliche Krankenversicherung auf diejenigen zu konzentrieren, die des sozialen Schutzes wirklich bedürfen. Dabei kann den freiwillig Versicherten mit Einkommen oberhalb einer bestimmten Grenze - mit ihren Familienangehörigen machen sie etwa 10 Mio. Personen aus durchaus die Eigenvorsorge in der privaten Krankenversicherung gemäß dem Subsidiaritätsprinzip zugemutet werden. Wenn diese Gruppe dennoch die gesetzliche Versicherung der privaten vorzieht, dann doch wohl nur, weil sie sich von der Solidargemeinschaft im Verhältnis zu den gezahlten Beiträgen größere Vorteile als von der PKV verspricht. Es kann jedoch nicht Aufgabe einer gesetzlich organisierten Sozialversicherung sein, Gutverdienern - womöglich noch zu Lasten der Solidargemeinschaft - Versicherungsschutz zum "Niedrigpreis" zu geben.

Deshalb bedarf die Mitgliedschaft der freiwillig in der gesetzlichen Krankenversicherung Versicherten grundsätzlich der Überprüfung. Die Einbeziehung dieser Gutverdiener 
in die PKV wäre systemgerecht. Eine Regelung, die das automatische Ausscheiden aus der GKV vorsieht, wenn das Gehalt des Versicherten eine bestimmte, deutlich über der Versicherungspflichtgrenze liegende Höhe überschreitet, hat es früher schon gegeben. Sie wird im übrigen in den Niederlanden heute in ähnlicher Form noch ohne Probleme praktiziert.

\subsection{Versicherungsfremde Leistungen}

Dem sozialen Schutzauftrag der GKV widersprechen auch Angebote, die nur für eine kleine Gruppe der gesetzlich Versicherten, nämlich die der besserverdienenden, freiwillig versicherten Mitglieder erreichbar sind. Dazu gehört etwa das zusätzliche Krankengeld, das zu nicht kostendeckenden Beiträgen von den Ersatzkassen angeboten wird. Diese Zusatzleistung sollte demnächst gesetzlich untersagt werden. Der Einsparungseffekt dürfte hier bei rund 160 Mio. DM jährlich liegen.

Gestrichen werden sollte auch die gesetzliche Ermächtigung zur Erhöhung des Sterbegeldes bis zum Doppelten. Allein die Begrenzung des jetzt bis zu 5.700 DM reichenden Sterbegeldes auf den einfachen Satz von 2.850 DM würde jährliche Ersparnisse von fast einer Mrd. DM bringen. Eine Begrenzung auf den der PKV von der Aufsichtsbehörde gesetzten Höchstsatz von 500 DM würde die Kassen gar um rund 1,7 Mrd. DM entlasten.

\subsection{Kostenerstattung ohne Pendeln}

Bedenklich ist nach Ansicht der PKV auch die dem Sachleistungsprinzip widersprechende angebotene wahlweise Kostenerstattung. Sie wird ebenfalls nur den Beziehern von Einkommen oberhalb der Jahresarbeitsverdienstgrenze angeboten. Mit dem Bundessozialgericht tritt auch die PKV für klare gesetzliche Regeln ein. Wenn den Kassen die Möglichkeit eines solchen Angebots gestattet wird, dann müssen sie zugleich veranlaßt werden, die Versicherten dauernd oder zumindest für eine längere Frist auf das eine oder andere System festzulegen. Das bisher praktizierte ständige Pendeln zwischen Kostenerstattung und Krankenschein führt zur Aushöhlung des Systems.

\subsection{Konzentration der Familienhilfe}

Die beitragsfreie Familienhilfe muß nach Auffassung der PKV den veränderten Familienstrukturen angepaßt werden. Da heute schon rund $25 \%$ aller Ehen kinderlos sind, muß die Frage gestellt werden, welchen Sinn in diesen Fällen noch die Familienhilfe haben kann. Wenn die rund 500.000 nicht erwerbstätigen Ehepartner ohne Kinder den halben Beitrag zu zahlen hätten, ergäbe allein dies eine Mehreinnahme von 1,5 Mrd. DM für die Kassen. Selbst wenn eine solche Beitragspflicht auf die freiwillig Versicherten beschränkt bliebe, würde noch ein beträchtlicher Personenkreis erreicht werden.

\subsection{Beamte in die $P K V$}

Für Beamte gibt es ein eigenes Sicherungssystem im Krankheitsfall durch Beihilfen des öffentlichen Dienstherrn. Daher bedürfen sie nicht des sozialen Schutzes durch die GKV. Die PKV hat sich verpflichtet, alle Beamten und deren Angehörigen zu erleichterten Bedingungen, insbesondere bei der Risikoprüfung, aufzunehmen. Der Gesetzgeber ist aufgefordert, die Entmischung der Systeme zu Ende zu führen. Die PKV ist als Versicherungsträger der Beamten auf Dauer zu den erforderlichen flankierenden Maßnahmen bereit. 


\subsection{Gezielte Ausweitung der Selbstbeteiligung}

Einen Beitrag zur Solidarität, worunter auch kostenbewußteres Verhalten der Versicherten zu verstehen ist, kann auch die gezielte Ausweitung der Selbstbeteiligung in der gesetzlichen Krankenversicherung leisten. Zwar ist die Selbstbeteiligung kein Wundermittel, sie ist aber dort angebracht, wo nicht der Arzt, sondern weitgehend der Patient selbst den Schlüssel für die Kosten in der Hand hält. Dabei ist in erster Linie an den Arzneimittelsektor zu denken. Hier hat die private Krankenversicherung gute Erfahrungen gemacht. Die jetzige, geringe Zuzahlung zu Arzneimitteln, die so gut wie keinen Steuerungseffekt hat, sollte durch einen spürbaren prozentualen Eigenanteil abgelöst werden. Kein geringerer als Goethe bemerkte hierzu schon in seinen Maximen und Reflexionen : "Wo der Anteil sich verliert, verliert sich auch das Gedächtnis".

\subsection{Private Pflegeversicherung steuerlich fördern}

In Zusammenhang mit einer am Subsidiaritätsprinzip orientierten Neuordnung des Krankenversicherungssystems ist auch die Bewältigung des Pflegerisikos zu sehen. Mit der steigenden Zahl alter Menschen wird die Absicherung im Pflegefall weiter an Bedeutung gewinnen. Die Lebensversicherer und privaten Krankenversicherer bieten Pflegeversicherungen an, die eine flexible und kostengünstige Abdeckung dieses Risikos ermöglichen. Die eigenverantwortliche Vorsorge für das Pflegefallrisiko sollte allerdings durch entsprechende steuerliche Rahmenbedingungen gefördert werden. Es erscheint dagegen nicht sinnvoll, auch die Befriedigung dieses neuen Bedarfs wiederum der Eigenvorsorge etwa in der Form zu entziehen, daß eine pflichtweise Pflegeversicherung im Rahmen der GKV eingeführt wird. Die Kassen würden weiter zusätzlich belastet und in ihren ohnehin sehr mühsamen Stabilisierungsanstrengungen unnötig behindert. Dagegen ließen sich durch eine Förderung der privatwirtschaftlichen Lösung ordnungs- und sozialpolitisch erwünschte Effekte erreichen, ohne den Sozialetat und die Lohnnebenkosten weiter aufzublähen.

\subsection{PKV setzt auf den verantwortungsbewußten Versicherten}

Die PKV setzt auf den Versicherten, der gewohnt ist, eigenverantwortlich und verantwortungsbewußt zu handeln und entsprechend mit den knappen Gütern des Gesundheitsmarkts umgeht. Wer deshalb eine echte Strukturreform mit mehr Markt will, muß auch mehr private Krankenversicherung wollen. Privatisierung tut also auch in diesem Bereich not. Deshalb sollte der Staat nicht unnötig - wie in der Vergangenheit geschehen das Betätigungsfeld der privaten Krankenversicherung einengen, sondern sie im Gegenteil nicht zuletzt im eigenen Interesse in ihrer Arbeit tatkräftig unterstützen. Hier liegt eine Chance auch für eine "Wende" in der Sozial- und Gesundheitspolitik. 